\title{
ANISOTROPY OF MAGNETORESISTANCE AND SHUBNIKOV-de HAAS OSCILLATIONS IN ORGANIC METAL $(\mathrm{ET})_{3} \mathrm{CuCl}_{4} \cdot \mathrm{H}_{2} \mathrm{O}$
}

\author{
S.I. Pesotskir ${ }^{a, c}$, A.E. Kovalev ${ }^{b, c}$, R.B. LyUbovsKiI ${ }^{a, c}$ AND N.D. KUShCH ${ }^{a}$ \\ ${ }^{a}$ Chernogolovka Institute of Chemical Physics RAS, 142432, Chernogolovka MD, Russia \\ ${ }^{b}$ Institute of Solid State Physics RAS, 142432, Chernogolovka MD, Russia \\ ${ }^{c}$ International Laboratory of High Magnetic Fields and Low Temperatures
} 53-529 Wroctaw, Poland

\begin{abstract}
Behavior of the magnetoresistance has been studied in organic quasi two-dimensional metal (ET) $)_{3} \mathrm{CuCl}_{4} \cdot \mathrm{H}_{2} \mathrm{O}$. Shubnikov-de $\mathrm{Haas}$ oscillations have been observed at the magnetic field directions near $\boldsymbol{H} \| \boldsymbol{c}^{*}$. The oscillations frequency at $\boldsymbol{H} \| \boldsymbol{c}^{*}$ equals $45 \mathrm{~T}$ that corresponds approximately to $1 \%$ of the first Brillouin zone cross-section. The angle dependence of the oscillations frequency allows one to imagine at least the part of Fermi surface in the form of cylinder with an axis along $c^{*}$.
\end{abstract}

PACS numbers: $75.90 .+w$

\section{Introduction}

In 1988 Shubnikov-de Haas (SdH) oscillations were found in quasi two-dimensional (Q2D) organic metals [1]. Fermi surface (FS) became directly accessible for these materials. Since that time a great interest to the behavior of organic conductors synthesized on the base of ET (bis-(ethylenedithio)-tetrathiafulvalene) and its derivatives in a magnetic field has been permanently increasing. At present fermiology of organic metals and superconductors appears as an individual, the most intensely developing trend in the physics of organic conductors. Such an interest is stimulated mainly by the peculiarities of FS topology of indicated compounds associated with a lowered dimensionality of their electron systems. The existence of these peculiarities particularly enabled one to observe the principally new phenomena, namely giant angular oscillations of a classical part of magnetoresistance (AMRO), attributed to the electron motion both in closed [2] and open [3] orbits. Most attention is now paid to the system of layered isostructural metals (ET) ${ }_{2} \mathrm{MHg}(\mathrm{SCN})_{4}$, where $\mathrm{M}=\mathrm{K}, \mathrm{Tl}, \mathrm{Rb}, \mathrm{NH}_{4}$, which possesses a structure of $\alpha$-type for a conducting layer [4, 5, 6]. FS for metals of this system consists of two sheets: a closed cylinder, characteristic of Q2D electron system and two corrugated planes, inherent in Q1D structures [7]. This results in the coexistence 
in some metals of this system of the effects characteristic of both Q1D (for example the transition to SDW state) and two-dimensional (except SdH oscillations) metals $[6,8]$. We report here the preliminary results of the investigation of magnetoresistance of Q2D organic conductor $\mathrm{ET}_{3} \mathrm{CuCl}_{4} \cdot \mathrm{H}_{2} \mathrm{O}$, which also possesses the structure of the conducting layer of $\alpha$-type. Crystalline structure of this compound appears as conducting layers of ET cations parallel to $a b$ plane and separated by dielectric $\mathrm{CuCl}_{4} \cdot \mathrm{H}_{2} \mathrm{O}$ anions from one another along $c$ direction. ET molecules are parallel to each other in a conducting layer thus defining $\alpha$-type for a conducting layer [9]. The temperature dependence of the resistance is metal-like for this compound from $300 \mathrm{~K}$ down to $0.3 \mathrm{~K}$ with no peculiarities [10].

\section{Experimental, results and discussion}

We have studied single crystals of the organic metal $\mathrm{ET}_{3} \mathrm{CuCl}_{4} \cdot \mathrm{H}_{2} \mathrm{O}$, appearing as plates with characteristic sizes of $1.5 \times 1.0 \times 0.1 \mathrm{~mm}^{3}$ with the smallest size corresponding to the direction $c^{*}$ perpendicular to the conducting layers. Conductivity measurements were carried out by using a common four-probe method at $330 \mathrm{~Hz}$ alternating current. Four measuring Pt wires were glued onto a crystal with a graphite paste in such a manner that a $300 \mu \mathrm{A}$ working current passed along the conducting layers. The contacts were ohmic within the whole temperature range. In magnetoresistance measurements a magnetic field was generated by a superconducting solenoid and reached $15 \mathrm{~T}$. A gadget was used, which enabled a sample rotation over an angle exceeding $360^{\circ}$.

Without a magnetic field the temperature dependence of resistance of the samples under study is metal-like. In this case the absolute value of the resistance changes from a sample to a sample and the sample studied in the magnetic field possesses a resistance approximately equal to $0.1 \mathrm{ohm}$ at $H=0$ and $T=1.45 \mathrm{~K}$. A magnetoresistance of $\mathrm{ET}_{3} \mathrm{CuCl}_{4} \cdot \mathrm{H}_{2} \mathrm{O}$ single crystal is positive at any field direction and is strongly anisotropic. Figure 1 displays an angular dependence of a resistance for the sample at $H=15 \mathrm{~T}$ and $T=1.45 \mathrm{~K}$. The minimal magnetoresistance value constitutes approximately $20 \%$ and the maximal one is more than $100 \%$. Field directions in which minimum and maximum of magnetoresistance are attained do not correspond to any crystallographic axis known for this crystal. At the same time magnetic field directions, so extremely important for layered structures as perpendicular and parallel to the conducting layers, are not reflected in the angular dependence of magnetoresistance (see Fig. 1). Such magnetoresistance behavior is not characteristic of the well-known Q2D organic metals and its origin requires more detailed investigation.

At the field direction $\boldsymbol{H} \| \boldsymbol{c}^{*} \pm 50^{\circ} \mathrm{SdH}$ oscillations periodic over a reverse field were firstly found in organic metal $\mathrm{ET}_{3} \mathrm{CuCl}_{4} \cdot \mathrm{H}_{2} \mathrm{O}$ crystal (see Fig. 2). Their frequency $F_{0}$ is equal to $45 \mathrm{~T}$ at the field direction $\boldsymbol{H} \| \boldsymbol{c}^{*}$ perpendicular to the conducting layers. At the field deviation from this direction $\mathrm{SdH}$ oscillation frequency increases according to $F=F_{0} / \cos \varphi$ law which is linear in the polar coordinate system, where $\varphi$ is the angle between field and $c^{*}$ directions (see inset in Fig. 2). Such behavior of the angular dependence of SdH oscillations frequency enables one to think of FS sheet responsible for SdH oscillations as a smooth cylinder with an axis along $\boldsymbol{c}^{*}$. In layered structures an interlayer interaction may 


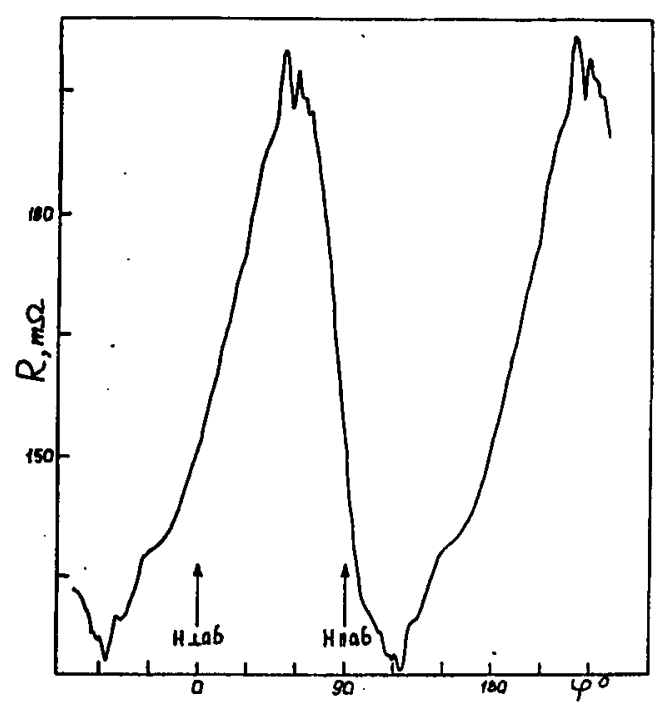

Fig. 1. Angle dependence of the resistance in the magnetic field in the plane parallel to $c^{*} . H=15 \mathrm{~T}, T=1.45 \mathrm{~K}$.

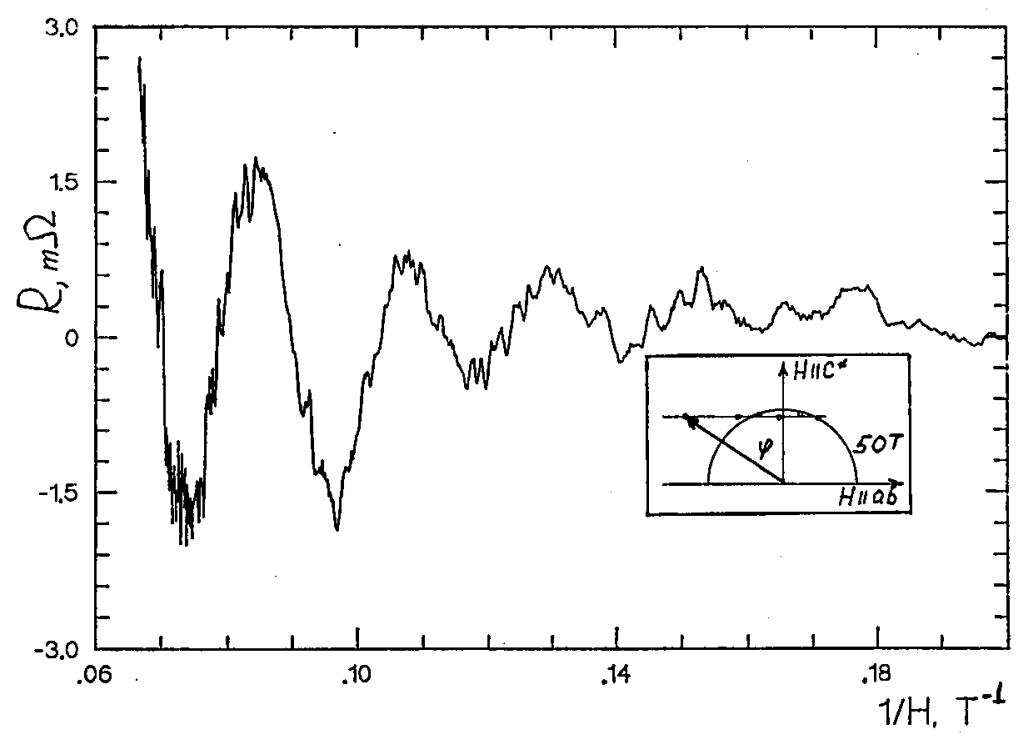

Fig. 2. Shubnikov-de Haas oscillations without a regular part of the magnetoresistance at $\boldsymbol{H} \| c^{*} . T=1.45 \mathrm{~K}$. In the inset the angle dependence of $\mathrm{SdH}$ oscillations frequency in the polar coordinate system.

result in a corrugated FS cylindrical sheet along $c^{*}$ direction [11]. However the main attributes of corrugation (SdH oscillation beats [11] and the occurrence of the angular oscillations of a classical part of magnetoresistance [2]) were not found 
in the sample studied. The cross-section of FS cylindrical sheet by $a b$ plane is approximately $1 \%$ of a corresponding cross-section of Brillouin zone. Such a small cross-section value in a conducting plane, provided that a conductivity value in investigated sample is typical for ET based organic conductors [10], enables the suggestion that FS sheet described constitutes only a part of the total FS in $\mathrm{ET}_{3} \mathrm{CuCl} \cdot \mathrm{H}_{2} \mathrm{O}$. Unfortunately theoretical calculations of FS are absent for this compound. Besides the presence of two $\mathrm{ET}_{3} \mathrm{CuCl} \cdot \mathrm{H}_{2} \mathrm{O}$ molecules in an elementary unit [9] evidences a more complex FS in it.

\section{Conclusion}

The main conclusion of this work is that at least a part of $\mathrm{FS}$ in $\mathrm{ET}_{3} \mathrm{CuCl} \cdot \mathrm{H}_{2} \mathrm{O}$ is found to appear as a smooth or weakly corrugated cylinder with an axis along the direction perpendicular to the conducting layers and with a cross-section in a conducting plane of approximately $1 \%$ of a corresponding one of Brillouin zone.

\section{Acknowledgment}

The authors express their gratitude to A.S. Borovik-Romanov, Ya. Klyamut and E.B. Yagubskii for the attention and the support of this work. The work was supported by the Russian Foundation of Fundamental Investigations (93-02-2384) and International Science Foundation RAS000.

\section{References}

[1] M.V. Kartsovnik, V.N. Laukhin, V.I. Nizhankovskii, A.A. Ignatiev, Pis'ma $Z h$. Eksp. Teor. Fiz. 47, 302 (1988) (in Russian).

[2] M.V. Kartsovnik, V.N. Laukhin, S.I. Pesotskii, I.F. Schegolev, V.M. Yakovenko, J. Phys. I (Paris) 2, 89 (1992).

[3] M.V. Kartsovnik, A.E. Kovalev, V.N. Laukhin, S.I. Pesotskii, J. Phys. I (Paris) 2, 223 (1992).

[4] H.H. Wang, K.D. Carlson, U. Geiser, W.K. Kwok, M.D. Vashon, J.E. Thompson, N.F. Larsen, G.D. McCabe, R.S. Hulscher, J.M. Williams, Physica C 166, 57 (1990).

[5] H. Mori, S. Tanaka, K. Oshima, G. Saito, Synth. Met. 42, 2013 (1991).

[6] N.D. Kushch, L.I. Buravov, M.V. Kartsovnik, V.N. Laukhin, S.I. Pesotskii, R.P. Shibaeva, L.P. Rozenberg, E.B. Yagubskii, A.V. Zvarikina, Synth. Met. 46, 271 (1992).

[7] H. Mori, S. Tanaka, K. Oshima, G. Saito, T. Mori, Y. Maruyama, H. Inokuchi, Bull. Chem. Soc. Jpn. 63, 2183 (1990).

[8] T. Sasaki, H. Sato, N. Toyota, Synth. Met. 41-43, 2211 (1991).

[9] R.P. Shibaeva, V.E. Kochetkov, L.P. Rozenberg, Kristallografija 36, 1447 (1991) (in Russian).

[10] L.I. Buravov, A.V. Gudenko, V.B. Ginodman, A.V. Zvarykina, V.E. Korotkov, N.D. Kushch, L.P. Rozenberg, A.G. Khomenko, R.P. Shibaeva, E.B. Yagubskii, Izv. Akad. Nauk SSSR Ser. Khim. N1, 223 (1990) (in Russian).

[11] M.V. Kartsovnik, P.A. Kononovich, V.N. Laukhin, R.B. Lyubovskii, S.I. Pesotskii, Zh. Eksp. Teor. Fiz. 97, 1305 (1990) (in Russian). 